\title{
Research on the Developmental Trend of Computer Music Composition under the Background of Popular Music and Aesthetics
}

\author{
Sui Wu \\ Hunan City University, \\ Yiyang,Hunan,413000 China
}

\begin{abstract}
In this paper, we conduct research on developmental trend of computer music composition under the background of popular music and aesthetics. In the process of the Chinese modern music history, popular music communication for the selection of music ontology, the audience for popular music transmission and delivery of the music ontology selection process fully the outline of Chinese modern society people's psychology and value concept of the construction of the trajectory and people value consciousness and change. Under this historical background, we propose novel computer music composition paradigm that will enhance and optimize the musical creation procedures.
\end{abstract}

Keywords- Developmental Trend, Computer Music, Composition, Popular Music and Aesthetics.

\section{Introduction}

Music is a kind of cultural phenomenon, but also associated with the physical properties of sound, the dual character of music refers to music at the same time, with this kind of the material and spiritual attribute. The essence of music creation and the spirit of human emotions, concepts such as activities to find a suitable for their own material carrier that extremely complex between spirit and the sound material of a kind of coordination double varieties of music composition law provisions on which the music directly. So, the research of history of composition law stipulation and should first start from the dual character of music. Music is the basic art of hearing. Auditory physiological tolerance, such as response to sound stimuli to heterogeneous, auditory memory of the music sound identification, the charge amount of complex information and caused by auditory function psychological feelings, etc., are all people to take the initiative to choose sound basic basis and the trade-offs. So, music's own movement by the threshold of auditory perception [1-3].

In the process of music creation, composer psychological dynamic is very complex and rich, and various psychological factors are intertwined interact and influence each other that could be listed as the following aspects. (1) Accumulated in the basic creation of the composer and creation activity, emotional memories and the feelings image memory is the main way of memory. Strong emotional memory can be concentrated, strengthen its wide experience of life, rich emotional memory and the feeling image memory more exquisite than normal people, emotional appeal more complex, more have stereo feeling. (2) Composer in the ocean of the life, perception, memory, the range is very wide. Both in the world of sound movement, and include the sound dynamic human psyche and all things of the world of the general form. (3) Composers in the perception of rich information, according to the morphological characteristics of music composing music movement, take the form of perception and memory object, modality, the structure of the state as the main contradiction. To our perception of the initiative, seize the overall structure of the object to complete the perceptual form of some abstract for creating the accumulation of valuable materials [4-5].

As an indispensable part of human life and music in many years ago has become the study of human cognitive and its potential mechanism is one of the effective tools of the brain. Tone of 
music is the organized people to express thoughts and feelings, which can reflect the real life an art, it is a complex cognitive process, by music speed, pitch, rhythm and mode and other measurable parameters, and the influence of the speed of the music is the most important characteristics of music emotional response. Degree is an inner experience of the rhythm feeling that is music composition according to the time relationship of each other to form the continuity of the whole. Music perception to cause the attention of many cognitive scientists, they put the music as a kind of the general-purpose product of cognitive architecture. In the figure one, we show the novel paradigm of computer music composition.

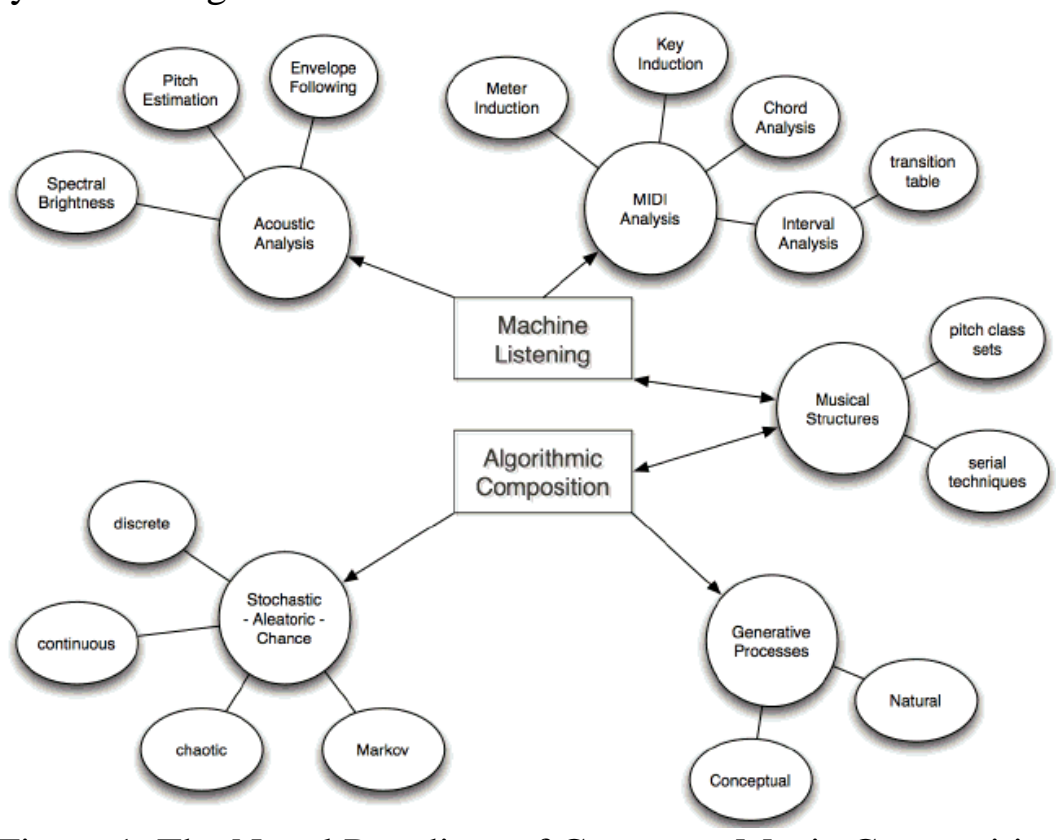

Figure 1. The Novel Paradigm of Computer Music Composition

In this paper, we conduct research on the developmental trend of the computer music composition under the background of popular music and aesthetics. Use computer powerful function, to represent the various digital signals for the recording and editing, music events sent to the electronic music synthesizer and makes it play out. For this set of creative and play the computer music system, we call it: computer music system. Computer music software is the soul of the computer music system. Music software is divided into five categories: sequence printing software, software, music sound editorial software, automatic accompaniment software and music education software. In the following parts and sub-sections, we will discuss the corresponding issues in detail.

\section{The Proposed Methodology and Perspective}

The Popular Music Concepts. Music since birth has an invisible spiritual power, it deeply into all aspects of general social life. All music activities are social activities process. The functions of music activities, reflecting the ways the contents, performance, ideas, attitudes, etc. are largely influenced by the certain social factors and constraints.

Popular music refers to a kind of easy to understand, relaxed and lively, easy to spread, with a large audience of music, serious music, classical music, and it is different from the traditional folk music. Say "popular music" is broad in this paper on the concept, widely circulated in the different historical period of mass music, popular and widely spread. Society forward at the same time, the corresponding 
social thought field will also change, this is a kind of inevitable rule. Music as part of the social spiritual culture, with respect to its source, thought essence, method and form a social function. Music is a reflection of human social life, social decided to the direction of the music culture. So, the music at the same time of contributed to the development of the society will be affected by the social culture and they both are interdependent, close relationship [6].

From the perspective of the popular music culture, popular music culture exists in the bottom of the society, reflects the social folkway and folk custom that is the picture of real life. Popular music in contemporary music culture has been a hot topic in life from the perspective of the sociology of the music systematically guides the healthy development to make public individual character that meet the demand of mass culture entertainment, popular music is to undertake social responsibility. From the view of macroscopic, the music education and even the whole education are set in social this big environment. Music transmission technology with each passing day, the public music activities are flawed, contact social life of music more and more closely, music education and social music culture is increasingly obvious, interactions between music education in new opportunities of development, also inevitably faces a challenge under the new situation.

We should fully expand their own taste as this is another requirement for all in music appreciation. That is to say, a viewer like only one type of music is no good. All kinds of the music, including the various periods of various genres, new and old, national and modern music to be listened to, and is as far as possible to listen to, without any prejudice to continuously expand their appreciation.

The Aesthetics Perspectiv of the Music. Music is not the specific space, this is the root of the music is different from other material properties. But should also be noted that any music is a sound wave continuation process and this process will take time. Anthropological research objects and the research object of aesthetics is still relevant and not the same, the former is the main research of music culture in all aspects, not merely to/not primarily aesthetic, the latter mainly studies on various aspects of music emotional needs and satisfaction, including specific related thoughts and behavior under the influence of the traditional culture and general history [7].

Form logic and common sense of law of excluded middle, we have reason to make the following inference by spiritual externalization of inner abstract thinking to form the aesthetic expression, the general form of the spirit of the requirements to the concrete bearing art contains, the logic of general exhibition will be guided by the spirit within to forward the art of emotional expression of external representation of the forces of the nature, namely the inherent nature of mental power forward to show emotion externalization of objectivity that is reflected from the following figure.

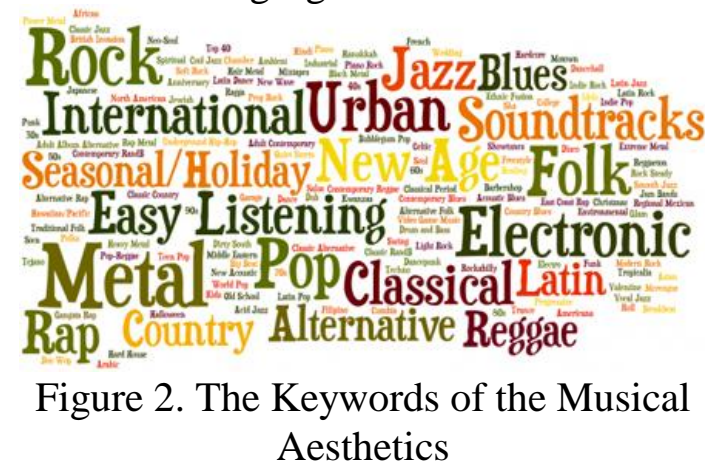

Of form and content on general multiple interpretations is still relatively intact involving multiple secondary perspective, and interpret the conclusion finally embodies readers to the work of the value judgment. (1) Analysis the purpose and task is different, each have each value as are beyond reproach. According to my understanding, the musicology analysis should be a higher level, has a professional comprehensive quality analysis as it is to investigate the artistic style of the works of music language and aesthetic features, and to reveal the social and historical 
content of music works. (2) At the same time of technical analysis, from the perspective of aesthetics, which further investigation of these techniques in humanities spirit connotation and inherent according to produce by these techniques. (3) Based on positivism as the main research ideas, the interpretation work mode, melody, harmony, melody structure, that tended to be integrated into the texture elements such as semiotics, rhetoric, learning in areas such as structural and image analysis technique.

The Music Composition Procedures. To some extent, music creation reflects the basic features of music art development. To traditional music is almost a reflection of music creation history, the object of study in music works and also mainly composers. The historical research methods may exist, but at least it shows: in the creation of the sound east across the entire music art practice of absolute important position. Modal of the structure of the perception and basic memory is mainly in perception object movement form at the same time, grasp the emotional tone and presented the form change, feel the power and tension in form and what the relationship between motivation and stress of emotion.

The form of music, with part of the law is or so by nature. Harmonic of the sound, sound, rhythm, structure of orderly and reflect in symmetry, balance, contrast, and unified aesthetic principles, has a gift for part actually also is the instinct of a man, that is human nature a kind of reflection, belong to the category of music material properties. The physiological characteristics of though in evolution, but relative to the development of human society, are much more slowly. This is what people often say that the music of the material basis of universality, identity depends. We are going to discuss here the history of the stipulation of composing techniques, as of course does not mean that all the physical attributes and change is slow, but people in real live music can often feel, or due to create conditions for different forms of each different, or for the development of society and constantly updated some skills, this is the social nature of music in a certain reflection on the composing techniques.

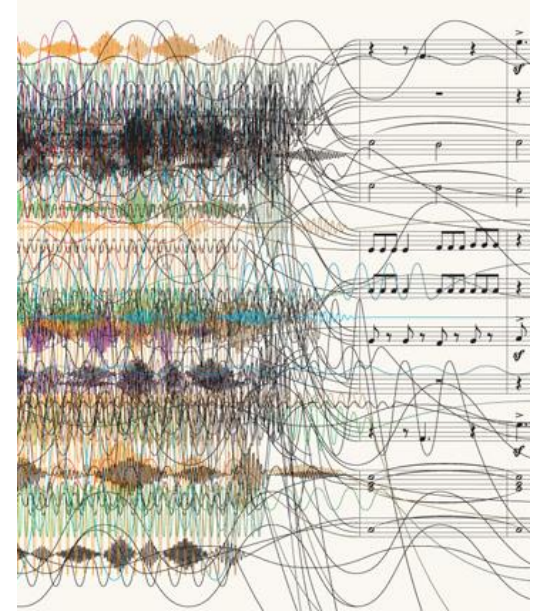

Figure 3. The Visualized Features of the Music Composition Procedures

The Computer Music Composition Paradigm. Recently, the more care about modern music production field, heat up suddenly, will find computer audio workstation at home and the abroad on magazine, newspaper, website, advertising and articles on computer audio workstation software and hardware more clearly, there are more and more people begin to talk about related topics.

The function of the computer teaching of music theory that deeper theoretical study has a good supporting role. Through good design by computer program to learn various skills of music, can you don't have to spend a lot of energy to various skills to master better, needless to say, this is of course a shortcut. Therefore, we summarize the corresponding techniques as the follows. (1) Can be used as the function of general tape recorder. Remember million function of computer can store notes from synthesizer or resting orders. When using synthesizer after playing a piece of music, need to repeat it again, at this time of the computer has long been the played signal stored just now. Then just press the button, the synthesizer will automatically double again as vivid as just 
playing on the keyboard. (2) Check that the function of music. MIDI is another function, can from the sound into a vivid and clear music, then, the score calibrated, the computer automatically by the synthesizer and then check the good music to play out through this function, printed music spectrum face clear, a high accuracy. (3) The function of acoustics can simulate the orchestra. Using a choreography good programs and high quality of the synthesizer, can simulate a pipe band sound or other ensemble sound effect can regulate the analog audio configuration and combination, and in a few minutes to listen to the effect of the computer to play sound system configuration [8].

Above all, really has the artistic expression of music animation is not only a pure mechanically converts audio visual, the creator for the understanding of music, thoughts, emotions, and many other factors differences often leads to a variety of different styles of visual forms. Traditional music visual creation because of his participation tend to have distinct emotional colors, and technology through the computer generated music visual animation doesn't become works of art and the key still needs creators thought and the emotion is blended in among them and send out.

\section{Conclusion}

In this paper, we conduct research on the developmental trend of the computer music composition under the background of popular music and aesthetics. Music is spread in human social intercourse activity interaction phenomenon of culture as is the process of sharing culture, and social values is transmitted, strengthening and acceptance process. In the process of the value of the music ontology spread, because the medium of participation, medium value along with the development, it has to do with music ontology value function and basic influence on people's psychology and value concept, influence people's value judgment and choice, will ultimately affect the building of the people value consciousness. Under this background, our paper combines the computer music concept to propose the novel perspectives of the issues that is meaningful.

\section{Reference}

[1] Huang, Chih-Fang, and Yun-Sheng Yeh. "Graphical interface-based automated music composition use among elementary school students." Musicae Scientiae 18.1 (2014): 84-97.

[2] Villani Jr, A. David. "An appraisal of the use of computer music notation software among selected high school music teachers." (2014).

[3] Peters, Nils, Trond Lossius, and Jan C. Schacher. "The spatial sound description interchange format: Principles, specification, and examples." Computer Music Journal 37.1 (2013): 11-22.

[4] Vincent, Tom, and Bill Martin. "Music, sounds, braille and speech - a multi-media computer for blind people." Equality and Diversity in Education 2: National and International Contexts for Practice and Research (2013): 220.

[5] Bryan-Kinns, Nick. "Mutual engagement and collocation with shared representations." International Journal of Human-Computer Studies 71.1 (2013): 76-90.

[6] Shang, Lin. "Enjoy your online music carefully: collective management of music copyrights in the USA." International Journal of Intellectual Property Management 8.1-2 (2015): 58-77.

[7] Wang, Cheng-I., and Shlomo Dubnov. "Guided music synthesis with variable markov oracle." 3rd International Workshop on Musical Metacreation, Raleigh, NC, USA. 2014.

[8] Wiberg, Mikael. "Interaction, new materials \& computing-Beyond the disappearing computer, towards material interactions." Materials \& Design 90 (2016): 1200-1206. 